\title{
Trapping a salt-dependent unfolding intermediate of the marginally stable protein Yfh1
}

\section{Bartolomé Vilanova ${ }^{1}$, Domenico Sanfelice ${ }^{2}$, Gabriel Martorell ${ }^{3}$, Piero A. Temussi ${ }^{4}$ and Annalisa Pastore ${ }^{2 *}$}

${ }^{1}$ Departament de Química, Universitat de les Illes Balears, Palma de Mallorca, Spain

2 Molecular Structure Division, National Institute for Medical Research, London, UK

${ }^{3}$ Serveis Científico-Tècnics, Universitat de les Illes Balears, Palma de Mallorca, Spain

${ }^{4}$ Department of Chemistry, Università di Napoli Federico II, Napoli, Italy

\section{Edited by:}

Sandra Macedo-Ribeiro, Instituto de

Biologia Molecular e Celular.

Portugal

Reviewed by:

Alfonso De Simone, Imperial

College London, UK

Neri Niccolai, University of Siena, Italy

\section{*Correspondence:}

Annalisa Pastore, Molecular

Structure Division, National Institute for Medical Research, MRC, The

Ridgeway, London NW7 1AA, UK

e-mail:apastor@nimr.mrc.ac.uk
Yfh1, the yeast ortholog of frataxin, is a protein of limited thermodynamic stability which undergoes cold denaturation at temperatures above the water freezing point. We have previously demonstrated that its stability is strongly dependent on ionic strength and that monovalent or divalent cations are able to considerably stabilize the fold. Here, we present a study of the folded state and of the structural determinants that lead to the strong salt dependence. We demonstrate by nuclear magnetic resonance that, at room temperature, Yfh1 exists as an equilibrium mixture of a folded species and a folding intermediate in slow exchange equilibrium. The equilibrium completely shifts in favor of the folded species by the addition of even small concentrations of salt. We demonstrate that $Y$ fh 1 is destabilized by a localized energetic frustration arising from an "electrostatic hinge" made of negatively charged residues mapped in the $\beta$-sheet. Salt interactions at this site have a "frustration-relieving" effect. We discuss the consequences of our findings for the function of Yfh1 and for our understanding of protein folding stability.

Keywords: cold denaturation, frataxin, metallo proteins, NMR, thermodynamic stability

\section{INTRODUCTION}

The yeast protein Yfh1 is a member of the highly conserved frataxin family that is present in most organisms from bacteria to primates (Pandolfo and Pastore, 2009). Yfh1 is a particularly fascinating protein amongst the frataxin orthologs. As all frataxins, it consists of a globular domain with an $\alpha \beta$ fold that is conserved in all species. However, despite the high degree of conservation, Yfhl is thermodynamically highly unstable at variance with other prokaryotic and eukaryotic members of the family, having the middle of its folding/unfolding transition around $35^{\circ} \mathrm{C}$, i.e., close to the optimal growth temperature of yeast (Adinolfi et al., 2004). It also undergoes another transition at low temperature, around $5^{\circ} \mathrm{C}$, being one of the very few known examples of a protein that undergoes cold denaturation at temperatures above water freezing point (Pastore et al., 2007). We have previously characterized in detail both the cold and heat unfolded states of Yfh1 and showed that they have similar residual secondary structure elements reminiscent of the architecture of the folded state, albeit with slightly different compactness (Adrover et al., 2012). We identified in the exquisite sensitivity of Yfh 1 to even small concentrations of salts, best if divalent or trivalent cations, an important factor to stabilize both unfolded states (Nair et al., 2004; Pastore et al., 2007; Sanfelice et al., 2014).

Thanks to the possibility of detecting both melting points, we reconstructed the whole stability curve that is, the plot of $\Delta \mathrm{G}$ as a function of temperature. The bell-shaped curve is narrow with a maximal stability in the range $18-23^{\circ} \mathrm{C}$ (Pastore et al., 2007; Martin et al., 2008; Sanfelice et al., 2014). By simple fitting, the curve allows to extract the thermodynamic parameters of the unfolding transitions and to measure the percentage of folded protein at the maximal stability temperature (Pastore et al., 2007; Martin et al., 2008; Sanfelice et al., 2014). This is only around 60-70\% (Pastore et al., 2007) a value which cannot solely be explained by the presence of an $\mathrm{N}$-terminal mitochondrial import signal (He et al., 2004; Karlberg et al., 2006). This observation suggests the possibility that Yfh1 exists in a partially unfolded state also at room temperature.

To explore this possibility, we focused on studying Yfh 1 at room temperature to ultimately understand the structural determinants that make Yfh 1 such a low stability protein and the bases of its salt sensitivity. Using NMR, we show here that Yfh1 exists in equilibrium between a folded species and a folding intermediate that has features in common with the cold and heat unfolded states. Even a small increase of the ionic strength is able to shift the equilibrium toward the folded species in agreement with the unusual sensitivity of Yfhl to salts. The presence of salts strongly affects a negatively charged patch on the $\beta$-sheet indicating that this is the main source of the energetic frustration of the Yfhl fold. Our results strongly suggest Yfhl to be another representative of metamorphic proteins whose fold depends on ionic strength.

\section{MATERIALS AND METHODS SAMPLE PREPARATION}

Recombinant S. cerevisiae Yfh1 was produced as previously described in details (Adinolfi et al., 2002; He et al., 2004) In short, the protein was expressed in Escherichia coli BL21-(DE3) 
cells grown at $37^{\circ} \mathrm{C}$, induced in $0.5 \mathrm{mM}$ IPTG for $2 \mathrm{~h}$. Cells were harvested by centrifugation and re-suspended in Tris- $\mathrm{HCl}$ buffer containing a complete EDTA protease inhibitor cocktail tablet (Roche) and lysed by 5 cycles of sonication. The soluble, overexpressed protein was purified by two ammonium sulfate precipitation steps with a $40 \%$ cut to precipitate contaminating proteins and a $65 \%$ cut to precipitate Yfh1. After dialysis, the protein was subjected to anion exchange chromatography using a Pharmacia Q-Sepharose column with a gradient to $1 \mathrm{M} \mathrm{NaCl}$, followed by a Pharmacia phenyl-Sepharose column with a decreasing $1 \mathrm{M}$ ammonium sulfate gradient. EDTA and salts were removed by dialysis prior to concentration of the protein. ${ }^{15} \mathrm{~N}$-labeled and ${ }^{15} \mathrm{~N},{ }^{13} \mathrm{C}$ double-labeled samples were produced by growing the bacteria in minimal medium using ammonium sulfate and glucose as the sole source of nitrogen and carbon. Final sample was stored in $20 \mathrm{mM}$ HEPES $2 \mathrm{mM}$ DTT pH 7.0.

\section{NMR SPECTROSCOPY}

${ }^{15} \mathrm{~N}$ - and ${ }^{15} \mathrm{~N},{ }^{13} \mathrm{C}$-labeled Yhf1 samples used for NMR studies $(\sim 0.5 \mathrm{mM})$ were in $20 \mathrm{mM}$ HEPES at $\mathrm{pH} 7.0$ and $2 \mathrm{mM}$ DTT, containing $5 \%(\mathrm{v} / \mathrm{v}) \mathrm{D}_{2} \mathrm{O}$. $\mathrm{NaCl}$ was added prior the experiments from a stock $(5 \mathrm{M})$ to a final concentration of $100 \mathrm{mM}$. Multidimensional NMR experiments were carried out at $298 \mathrm{~K}$ on a Bruker Avance spectrometer operating at 600 and $700 \mathrm{MHz}$ proton frequency and equipped with tripleresonance cryo-probe. The assignment of the backbone resonances of Yfh1 were accomplished by recording and analyzing HNCACB, CBCA(CO)NH, HNCO, and HBHACONH triple resonance datasets (Sattler et al., 1999). Data processing and analysis were performed using the nmrPipe (Delaglio et al., 1995), CARA (Keller, 2004), and NMRviewJ (Johnson and Blevins, 1994) software packages, respectively. Water was suppressed by the Watergate pulse sequence (Piotto et al., 1992). Proton chemical shifts were referenced to the water signal fixed at $4.7 \mathrm{ppm}$. ${ }^{13} \mathrm{C}$ and ${ }^{15} \mathrm{~N}$ chemical shifts were referenced indirectly using the ${ }^{1} \mathrm{H}, \mathrm{X}$ frequency ratios of the zero-point (Wishart et al., 1995). Secondary structure propensity (SSP) scores were calculated using the SSP software (Marsh et al., 2006). $\psi$ and $\varphi$ angles (Figure S1) were evaluated using Talos+ (Shen et al., 2009). The assigned chemical shifts of Yhf1 at $298 \mathrm{~K}$ are deposited in BioMagResBank (http://www.bmrb.wisc.edu) under accession number 19991.

\section{ROSETTA MODELING}

Structures of Yfh1 were calculated using chemical shift-based Rosetta (Rosetta 3.4) using a standard protocol (Ramelot et al., 2009) and incorporating backbone $\mathrm{N}, \mathrm{H}^{\mathrm{N}}, \mathrm{C}, \mathrm{C}^{\alpha}, \mathrm{H}^{\alpha}$, and $\mathrm{C}^{\beta}$ chemical shifts. After initial testing, the first 10 residues (from M51 to V61) of the intrinsically unfolded N-terminus were excluded from the calculation. The calculations generated 10000 all-atom models, and the 1000 low-energy models were extracted and further rescored against chemical shifts. The final 10 lowest energy structures were chosen as representative of the calculation. Pdb 2 ga5 and $2 \mathrm{fql}$ were excluded from the fragment search in order to have an unbiased calculation.

\section{RESULTS \\ AT ROOM TEMPERATURE THE FOLDED STATE IS IN EQUILIBRIUM WITH A FOLDING INTERMEDIATE}

The HSQC NMR spectrum of freshly produced and desalted Yfh1 at $23^{\circ} \mathrm{C}$ is overall well dispersed and typical of a well folded protein (Figure 1A, black). This is the temperature of maximum stability ( $T s)$ at these solution conditions, as extracted from its full stability curve (Sanfelice et al., 2014). However, closer inspection reveals the presence of more resonances than those expected for the sequence. In addition to the main peaks, several less intense resonances are observed, which are in the region of the spectrum expected for resonances of residues in a random coil conformation. The presence of these peaks was observed in independently produced batches of protein, suggesting that they account either for reproducible contaminants or for minor populations of the same protein.

\section{SALT STABILIZES THE FOLDED STATE OF Yfh1}

To test the hypothesis that the additional resonances could come from an unfolded or partially unfolded intermediate of Yfh1 in co-presence with the folded one, we added salt since we had earlier realized that salts have an appreciable effect on the protein stability (Adinolfi et al., 2004). A recent quantitative study on the effect of several salts revealed that divalent cations bind specifically at micromolar concentrations, greatly stabilizing the folded species, but even buffers containing monovalent cations induce sizeable stabilization at a relatively modest ionic strength (Sanfelice et al., 2014). By addition of $100 \mathrm{mM} \mathrm{NaCl}$, we observed complete and quantitative disappearance of the additional resonances and a minor shift of some resonances according to the change of experimental conditions (Figures 1B-E). The effect is fully reversible: desalting leads to reappearance of the extra peaks (Figure 1C).

These results strongly suggest that the folded and the intermediate forms are in mutual exchange so that the latter species disappears when the protein is stabilized. Comparison among the spectra at 0,23 , and $45^{\circ} \mathrm{C}$ suggests an overall similarity of the chemical shifts. There is not, of course, a precise coincidence, partly because of temperature dependence of all resonances (Figure 2) (Adrover et al., 2010). The number of resonances observed at $25^{\circ} \mathrm{C}$ for the intermediate is overall a relatively small number (ca. 30 resonances) suggesting a partial unfolding only in this region.

\section{SPECTRAL ASSIGNMENT PROVIDES IMPORTANT INFORMATION ON THE FOLDED STATE}

To identify the residues in exchange, we tried to assign the resonances of the minor species. For consistency with previous NMR studies (Cook et al., 2006) the assignment was performed at $25^{\circ} \mathrm{C}$, a condition only marginally different from $T_{S}$. However, we realized immediately that the available assignment of the spectrum of folded Yfh1 (BMRB accession code: 6356) may be partially incorrect as it traces resonances both from the folded species and the folding intermediate. Exploiting recent findings on the influence of salts on the stability of Yfh1 (Sanfelice et al., 2014), we assigned the spectrum anew both at nominal "zero ionic strength" and at moderate ionic strength $(100 \mathrm{mM} \mathrm{NaCl})$ (Figure 3). 


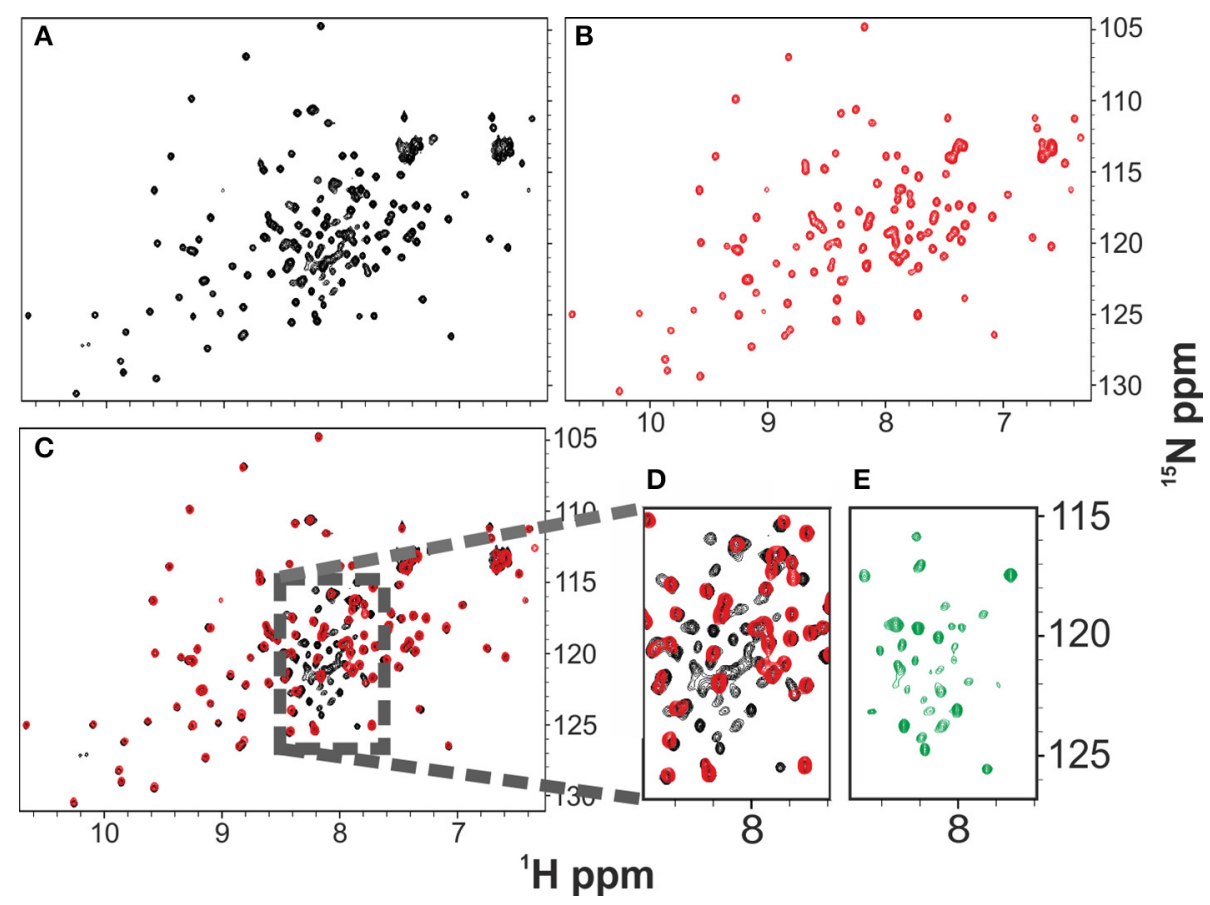

FIGURE 1 | Comparison of the ${ }^{1} \mathrm{H}^{15} \mathrm{~N}$ HSOC NMR spectra of $\mathrm{Yfh} 1$ in the presence (red) and in the absence (black) of salt. NMR experiments were acquired at $25^{\circ} \mathrm{C}$. (A) HSQC spectrum of freshly produced Yfh1 in $20 \mathrm{mM}$ HEPES buffer, pH 7.0. (B) HSQC spectrum of Yfh1 in $20 \mathrm{mM} \mathrm{HEPES} \mathrm{buffer}$ with the addition of $100 \mathrm{mM} \mathrm{NaCl}, \mathrm{pH} 7.0$. (C) Overlay of spectra $(\mathbf{A}, \mathbf{B})$ showing that many peaks of the central region (typical for unfolded protein peaks) disappear after salt addition. (D) Zoom of the central region between 8.5 and $7.5 \mathrm{ppm}$. (E) Zoom of the central region between 8.5 and $7.5 \mathrm{ppm}$ after subtraction of the red peaks: the remaining peaks coincide with those previously assigned to the unfolded species (Adrover et al., 2010, 2012).
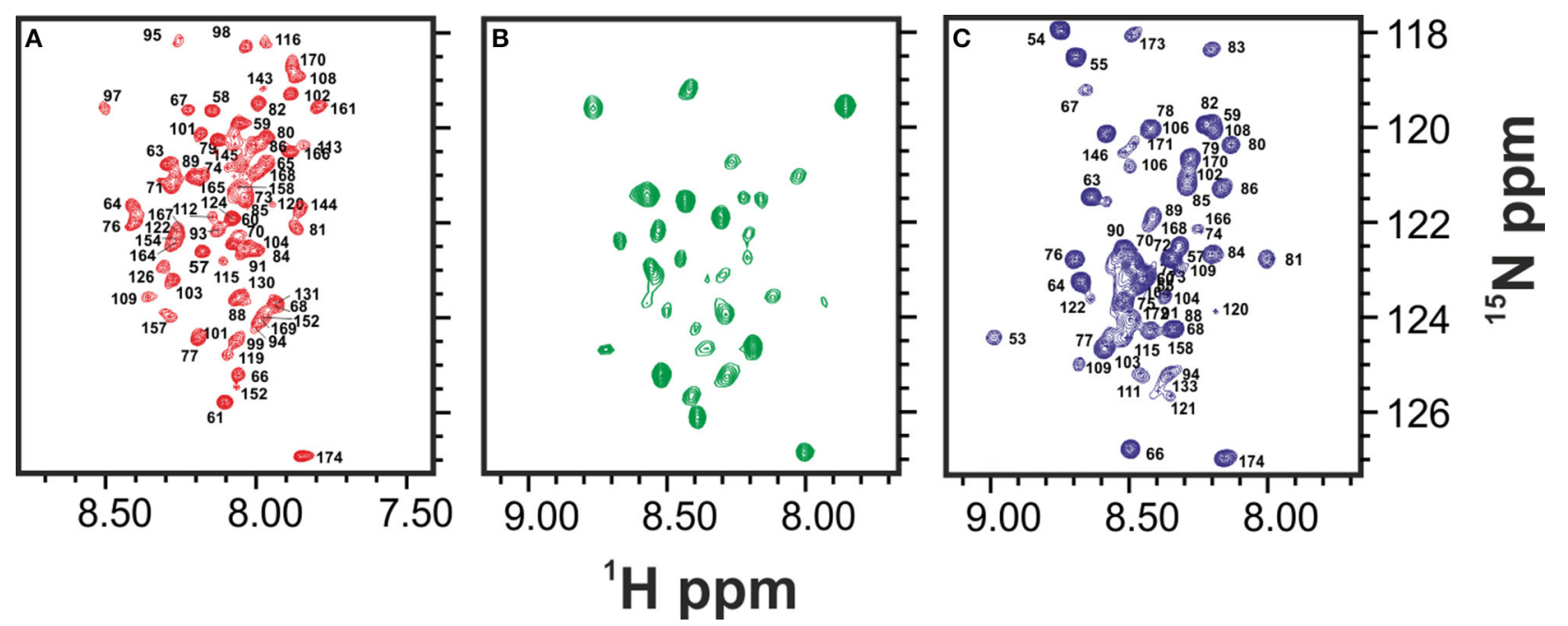

FIGURE 2 | Comparison of the HSOCs of the unfolded species of Yfh1 at high (red), low (blue), and room temperature (green). (A) HSQC spectrum of $\mathrm{Yfh} 1$ in $20 \mathrm{mM}$ HEPES buffer, $\mathrm{pH} 7.0$ at $45^{\circ} \mathrm{C}$ (Adrover et al.,
2012). (B) HSQC spectrum of $Y$ fh 1 in $20 \mathrm{mM}$ HEPES, pH 7.0 at $25^{\circ} \mathrm{C}$. (C) $\mathrm{HSQC}$ spectrum of $\mathrm{Yfh} 1$ in $20 \mathrm{mM}$ HEPES buffer, $\mathrm{pH} 7.0$ at $0^{\circ} \mathrm{C}$ (Adrover et al., 2010).
The new assignment differs from the old one by $12 \%$ for $\mathrm{N}$, up to $31 \%$ for $\mathrm{H} \alpha$ (see also Figure 4) and is virtually complete having assigned also the aromatic side chains (but for H106) which were missing in the previous assignment. Interestingly, the major differences between the two assignments are observed in the middle of the sequence, between residues 113 and 125 which correspond to a $\beta$-sheet region (Figure 4). These residues were correctly traced in the sequence but incorrectly assigned because they come from the same residues but in the unstructured form. 


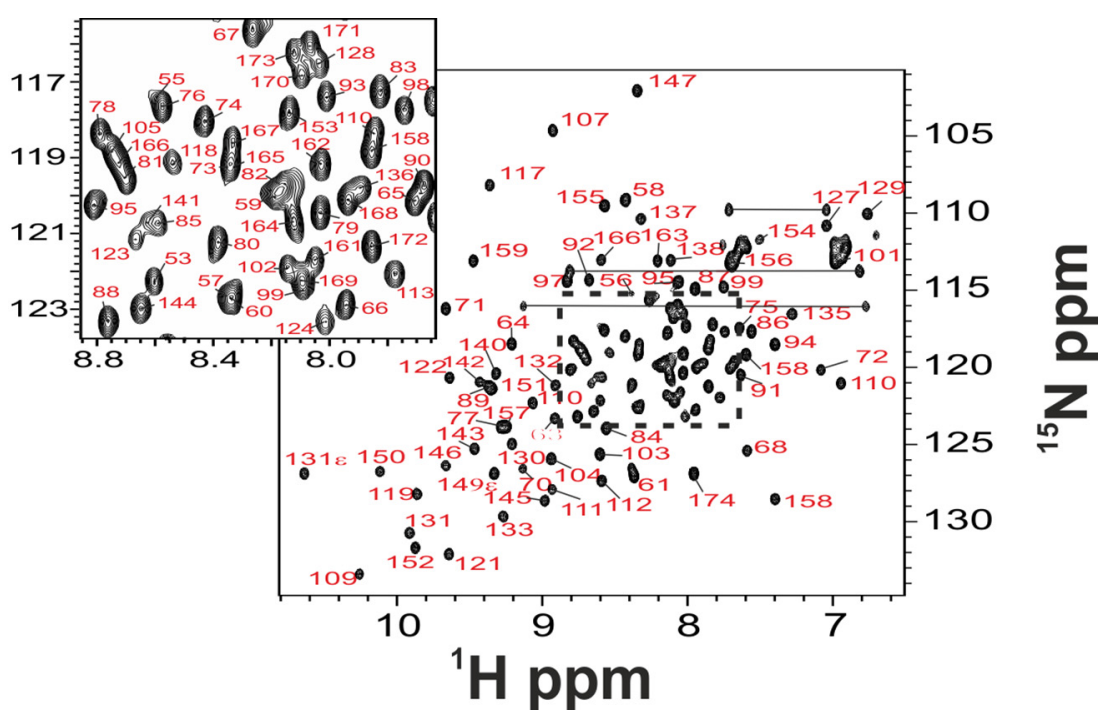

FIGURE 3 | Full backbone NMR assignment of folded Yfh1. NMR experiments on Yfh1, in $20 \mathrm{mM}$ HEPES buffer with the addition of $100 \mathrm{mM}$ NaCl, pH 7.0, were acquired at $25^{\circ} \mathbf{C}$. The inset shows assignment details in the region where there is maximum overlap of peaks typical of the unfolded species.
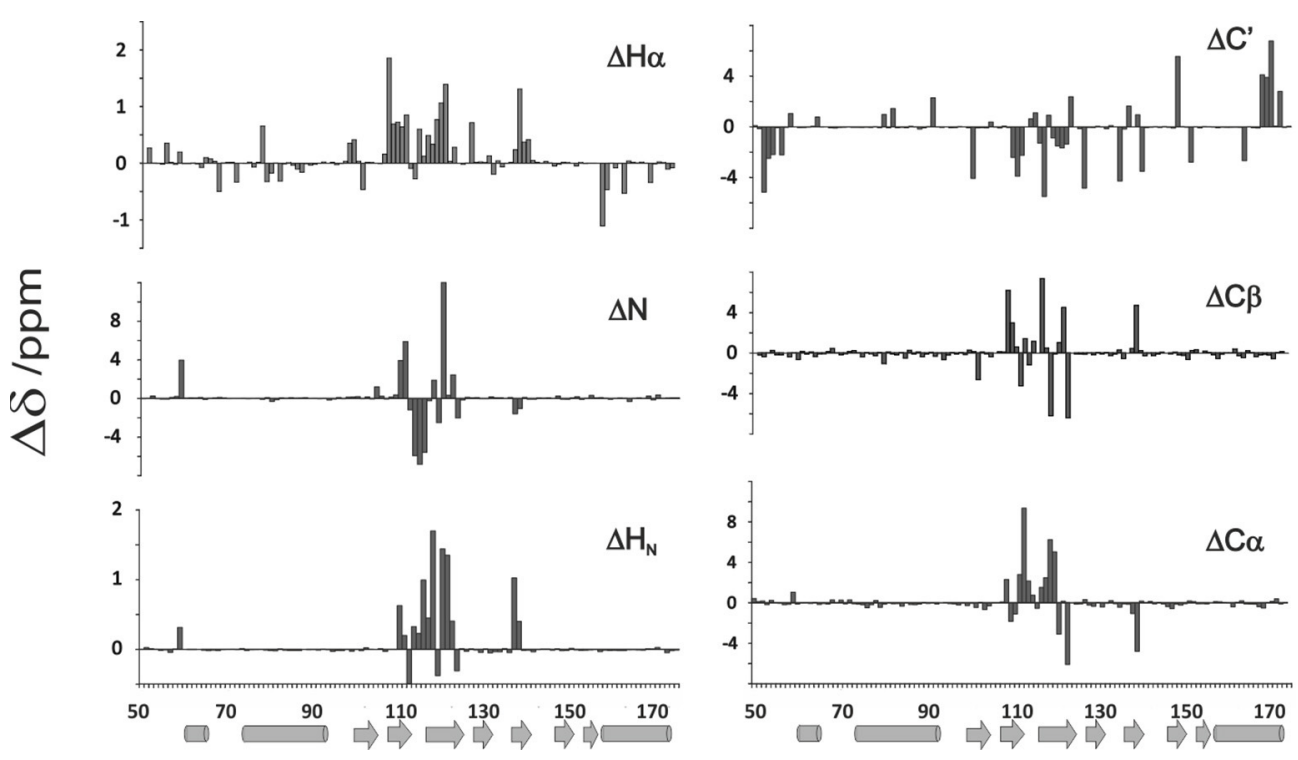

$\Delta \mathrm{N}$

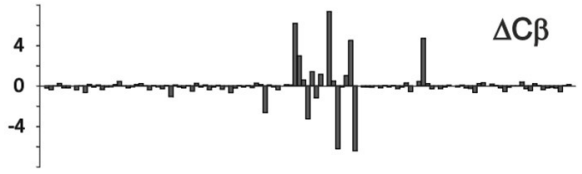

FIGURE 4 | Comparison of newly assigned chemical shifts with those previously assigned to yeast frataxin (He et al., 2004; Cook et al., 2006) (BMRB accession code: 6356). The bar graphs report differences between the two assignments. Salts have a strong influence on the residues going from 113 to 125 of the matured Yfh1. Percentage of residues presenting a $\delta / p p m$ absolute variation larger than $0.2(\Delta \delta \mathrm{ppm})$ are $13.0 \% \mathrm{HN}, 12.2 \% \mathrm{~N}, 26.8 \% \mathrm{H} \alpha, 16.3 \% \mathrm{C} \alpha, 17.1 \% \mathrm{C} \beta$, and $28.5 \% C^{\prime}$.

\section{STRUCTURE DETERMINATION OF Yfh1 IN SOLUTION}

As a validation of the newly determined assignment, we used the chemical shifts to model the structure of Yfh1 using the CSRosetta software (Qian et al., 2007; Shen et al., 2008; Ramelot et al., 2009). This method is based on an empirically optimized procedure that selects protein fragments from the Protein Data Bank, in conjunction with the standard ROSETTA Monte Carlo assembly. It allows the structural use of chemical shifts even in the absence of NMR restraints, resulting in significant improvements in structure quality and root mean square deviations (r.m.s.d.) with respect to the crystal structures. When applied to the new assignment of Yfhl the method converged effortlessly into a structure that shows all the features of the frataxin fold having two helices packing against a five strands $\beta$-sheet (Figure 5). Convergence improves by cutting off the first 10 residues which seems to be unfolded. Interestingly, the Rosetta software did not find convergence when using the previous assignment (Cook et al., 2006). 
A

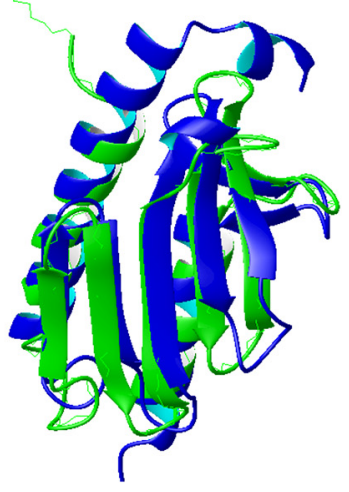

B

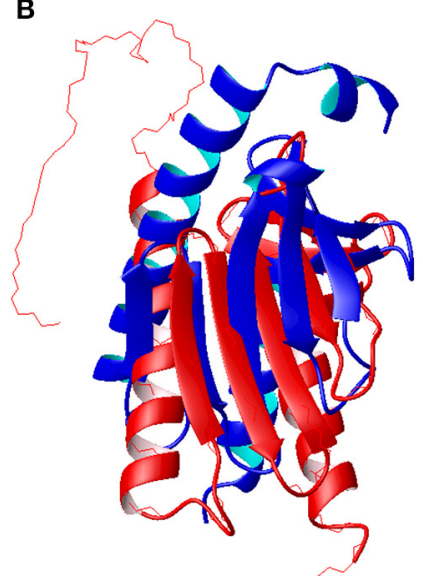

FIGURE 5 | Comparison of the molecular models of Yfh1. (A)

Superposition of the Rosetta model (blue ribbon) with the $x$-ray structure of a mutant Yfh1 (green ribbon, pdb id 2fql). (B) Superposition of the Rosetta model (blue ribbon) with the previous NMR structure (red ribbon, pdb id 2ga5). Molecular models, represented as ribbons, were built with MOLMOL (Koradi et al., 1996).

The r.m.s.d. with the crystallographic structure (pdb id 2fql) is $2.05 \AA$ (Figure 5A), whereas the corresponding r.m.s.d. with the previous NMR structure (pdb id 2ga5) is $3.05 \AA$ (Figure 5B). The major differences between the published structures and the Rosetta model, besides the different orientation of secondary structure elements in the previous NMR structure, are located at the $\mathrm{N}$ terminal. The NMR structure and the Rosetta model have same length of helix 1 while the crystal structure is two turns shorter, partly because it refers to a Y73A mutant designed to render the $\mathrm{N}$-terminal more flexible. The solution structure has also a 16aa long unstructured $\mathrm{N}$ terminal that is anchored to helix one; in the other two structures this region, although very flexible, presents a small helix.

\section{CHARACTERIZATION OF THE FOLDING INTERMEDIATE}

To characterize further the unfolded species and understand its degree of unfolding, we used the SSP indices, which combine different chemical shifts into a single secondary structure propensity score (Marsh et al., 2006) (Figure 6A). The positive and negative values ought to correlate with the helical and $\beta$-sheet elements

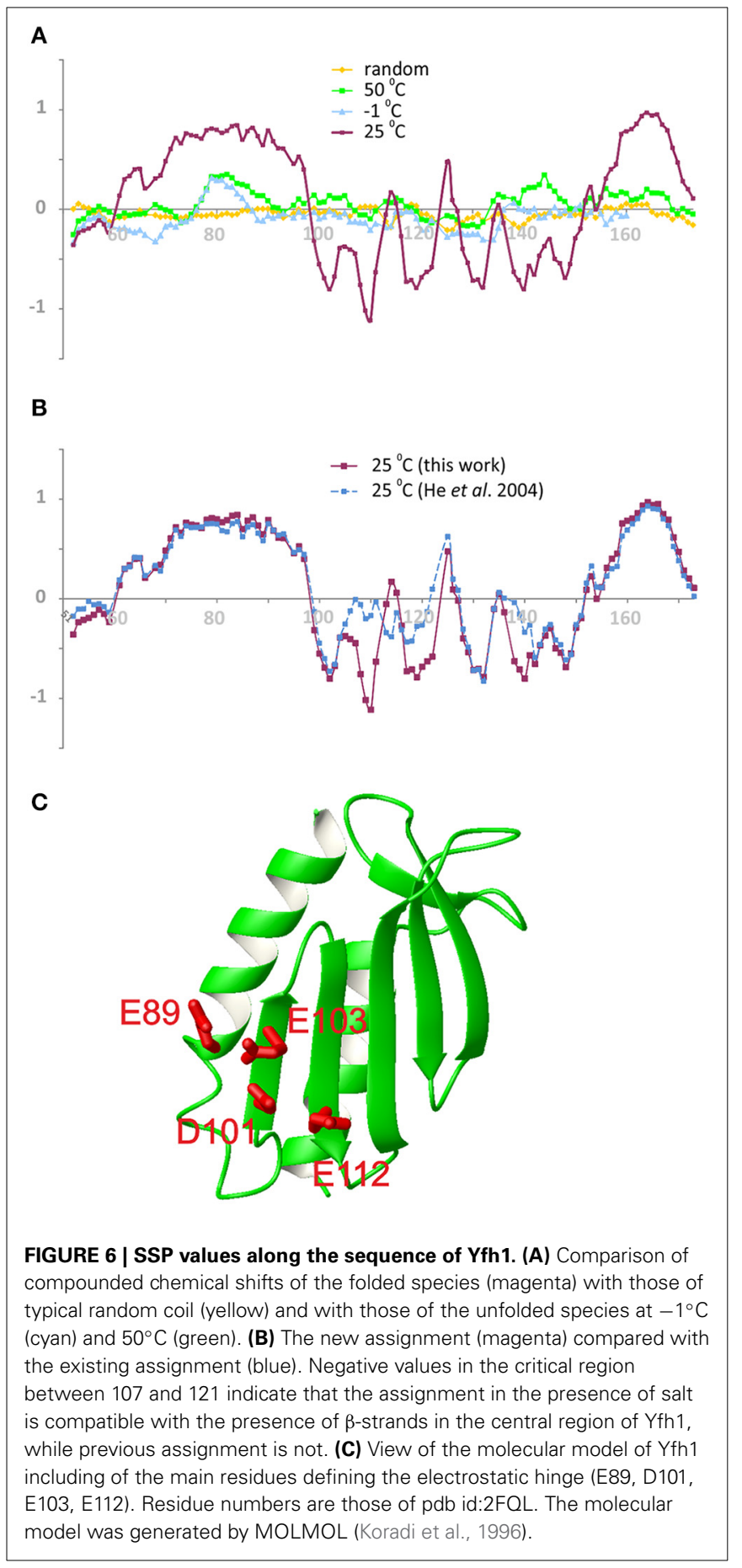

respectively, whereas values around zero indicate a random coil conformation. The values of the folded species correlate well with what observed in the frataxin family. The chemical shifts of the folding intermediate have, instead, a clear departure from the secondary structure observed for the folded species between 107 and 121 (Figure 6B). The values are closer to the random coil values expected for this sequence. Interestingly, the SSP profile in this region is more similar to that observed in both the high and low temperature unfolded states (Adrover et al., 2010, 2012). 
The analysis of the two assignments is nicely complemented by the use of a newly developed program, $\delta 2 \mathrm{~d}$ (Camilloni et al., 2012), which in addition to canonical elements of secondary structure yields also polyproline II propensities for each residue. The graphs of Figure 7 show that the divergence between the assignment of the present work and the previous one (BMRB accession code: 6356 ) is even more pronounced. The region where the divergence occurs maps onto a highly negatively charged region which contains a network of spatially nearby uncompensated negative charges, originating from the side chains of E89, E103, D101, and E112 (Figure 6C). According with the presence of a highly frustrated region, the extent by which this region is influenced by salt is clearly observable in the SSP plot. The corresponding residues in other tested frataxin orthologs are not equally charged, correlating with their higher stabilities and with the ability of Yfh1 to undergo cold denaturation. These data thus strongly suggest the presence of energetic frustration due to the high density of negatively charged residues as a major cause of Yfh1 instability.

\section{DISCUSSION}

The stability of globular proteins reflects the subtle balance of several different stabilizing and destabilizing contributions. Usually, we assume that proteins have evolved to optimize their stability with respect to the environmental constraints which they are active in, among which temperature. Proteins from mesophilic organisms, for instance, are usually stable around room temperature and over a relatively large range of temperatures (Razvi and Scholtz, 2006). There are however, examples of globular proteins which are just about stable also at room temperature. Yfh1 is not the only example of such behavior: while the fold of many globular proteins is unaffected by changes in experimental conditions within a large temperature range, other proteins, such as the well characterized U1A (Ternström et al., 1999) and azurin (Zong et al., 2007), have a broad transition energy barrier which leads to what we could call a "malleable" structure. This behavior was suggested to arise from the presence of strained energetics in the native state ensemble which leads to energetic frustration (Rimratchada et al., 2014). The systems that experience such frustration may provide unique information on both the early and late events of folding, as well as on the continuum of states in between. They are thus of particular interest from an experimental perspective.

For marginally stable proteins, a fascinating question is whether their behavior is evolutionary selected and beneficial for their functions or if other factors might intervene to stabilize the proteins so that they do not usually experience in vivo the conditions under which they are unstable. To understand the determinants of Yfh1 instability, we have analyzed in detail the behavior of the protein at room temperature. In previous studies we identified both the length of the C-terminus (Adinolfi et al., 2004) and salts (Sanfelice et al., 2014) as important factors that influence the stability of Yfh1 to the point that most salts increase considerably the unfolding point. Here, we have proven that Yfh1 exists in solution at room temperature as an equilibrium mixture of a folded and a partially unfolded species and that the equilibrium can be influenced by variations of the ionic strength. At low

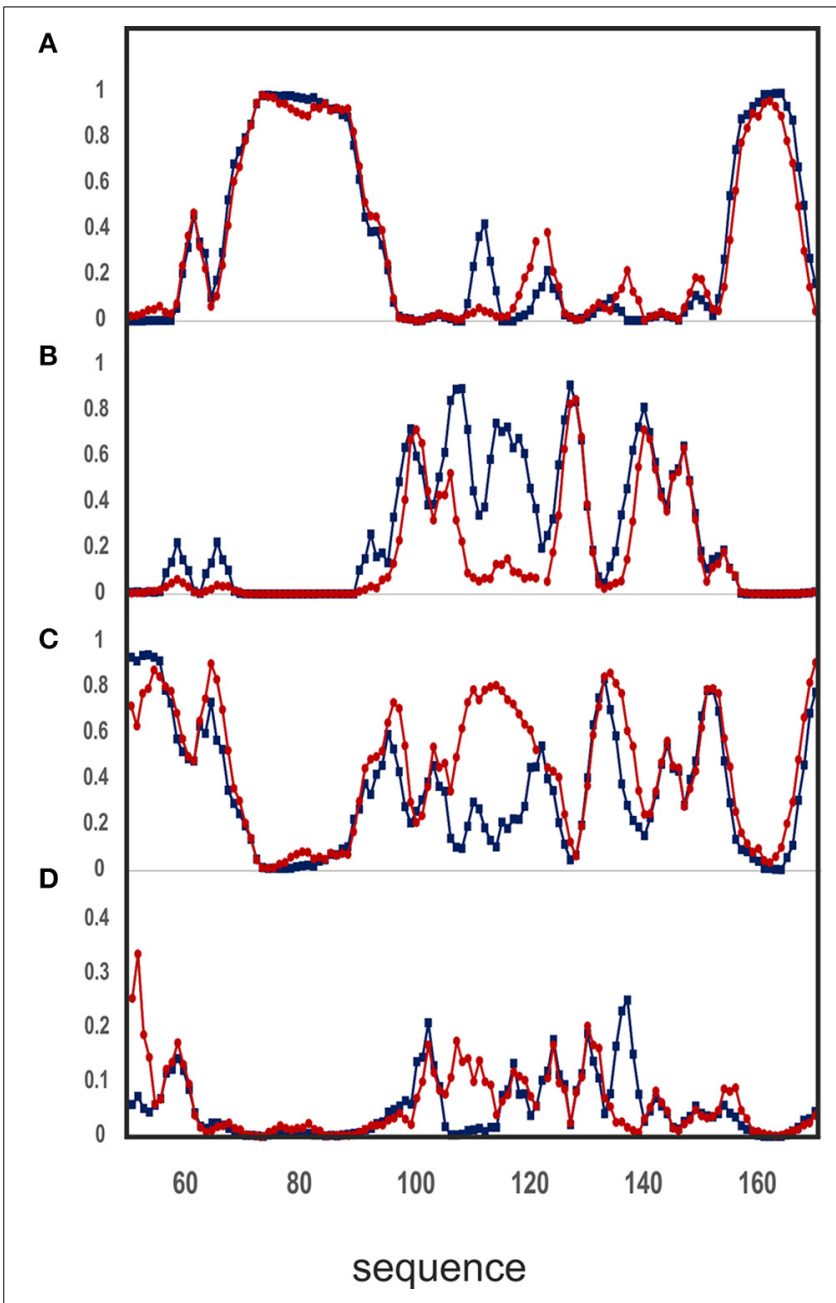

FIGURE 7 | Analysis of chemical shifts according to the $82 \mathrm{D}$ software, version 1.2. Comparison of the NMR assignment of the backbone atoms for Yfh1 from this work (blue) and that previously reported (BMRB accession code: $6356 /$ red) against the $\delta 2 D$ database yields the secondary structure propensity of the protein. (A) Helix propensity. (B) Extended beta sheets propensity. (C) Random coil propensity. (D) Polyproline II propensity. Residue numbers are those of pdb id:2FOL.

ionic strengths, the two species are present with an approximately 70-to-30 folded/unfolded ratio in favor of the folded conformer. The equilibrium is completely shifted toward the folded species when the ionic strength is increased. The intermediate has a spectrum broadly reminiscent of the spectra observed in the unfolded states at high and low temperatures (Adrover et al., 2010, 2012; Sanfelice et al., 2014). We believe that this is currently the only example that permits a comparison between NMR spectra of genuine unfolded or partially unfolded species at three different temperatures for a non-intrinsically unfolded protein.

Further, we mapped the effects that control the position of the folded vs. unfolded conformational equilibrium of low ionic strength Yfh1 to a specific region of the protein. Through the chemical shifts of the folding intermediate, we have experimentally identified the region of the protein where unfolding occurs 
(this study). The experimental evidence points toward a small cluster of negatively charged residues spatially close in the structure as an important source of energetic frustration. They comprise D101 and E103 in the $\beta 1$ strand and E112 in $\beta 2$ and form an "electrostatic hinge." An important contribution of the $\beta$-sheet in destabilization is also fully consistent with previous indications that this region is the most affected by addition of mono- and di-valent cations with the consequence of stabilizing the protein up to rendering the stability curve of the protein much more shallow and to shifting cold denaturation to much lower temperatures (Sanfelice et al., 2014). This is explainable considering that proteins are polymers with many degrees of conformational freedom whose internal repulsive energetic interactions are typically screened to small distances. However, the residues of Yfh1 are so close in space that can only be efficiently screened by counterions. These residues are semi-conserved in other members of the frataxin family correlating well with the somewhat unusual instability of Yfh 1 as compared to other orthologs (Sanfelice et al., 2014).

Our results have important implications. On a practical side, they stress the importance of salt to carry out in vitro functional studies aimed at characterizing the native function of proteins and specifically of Yfh1. More importantly, they indicate the importance of cation binding for the fold and function of Yfh1. In facts, the residues involved are among those identified to participate in iron binding and in recognition of one of the important natural partners of frataxin, the desulfurase Nfs1 (Adinolfi et al., 2009). We can also predict that the destabilizing interactions are where unfolding starts and that they are an important factor for observing cold denaturation of this protein. It will be interesting in the future to mutate some of these residues to release the effect and analyze how this may affect fold stability.

Finally, it is in order to draw a comparison between Yfh 1 with another prototypical example of marginally stable proteins stabilized by salts: this is the IscU protein which, interestingly, shares with Yfhl the rather acidic isoelectric point (around $\mathrm{pH} 4.5$ ), the intrinsic marginal stability that leads to cold denaturation at detectable temperatures and the existence of an equilibrium between a folded and a partially unfolded species in the absence of stabilizing cations (Iannuzzi et al., 2014). This analogy suggests that these are members of a new family of proteins in which energetic frustration due to destabilizing electrostatic interactions plays an important role in folding. It will be thus interesting to identify the causes that determine the properties of $\mathrm{IscU}$ at the residue level.

\section{ACKNOWLEDGMENTS}

The work was supported by MRC (U117584256).

\section{SUPPLEMENTARY MATERIAL}

The Supplementary Material for this article can be found online at: http://www.frontiersin.org/journal/10.3389/fmolb. 2014.00013/abstract

\section{REFERENCES}

Adinolfi, S., Iannuzzi, C., Prischi, F., Pastore, C., Iametti, S., Martin, S. R., et al. (2009). Bacterial frataxin CyaY is the gatekeeper of iron-sulfur cluster formation catalyzed by IscS. Nat. Struct. Mol. Biol. 16, 390-396. doi: 10.1038/nsmb.1579
Adinolfi, S., Nair, M., Politou, A., Bayer, E., Martin, S., Temussi, P. A., et al. (2004). The factors governing the thermal stability of frataxin orthologues, how to increase a protein' stability. Biochemistry 43, 6511-6518. doi: 10.1021/ bi036049+

Adinolfi, S., Trifuoggi, M., Politou, A., Martin, S., and Pastore, A. (2002). A structural approach to understanding the iron-binding properties of phylogenetically different frataxins. Hum. Mol. Genet. 11, 1865-1877. doi: 10.1093/hmg/11.16.1865

Adrover, M., Esposito, V., Martorell, G., Pastore, A., and Temussi, P. A. (2010). Understanding cold denaturation, the case study of Yfh1. J. Am. Chem. Soc. 132, 16240-16246. doi: 10.1021/ja1070174

Adrover, M., Martorell, G., Martin, S. R., Urosev, D., Konarev, P. V., Svergun, D. I., et al. (2012). The role of hydration in protein stability, comparison of the cold and heat unfolded states of Yfh1. J. Mol. Biol. 417, 413-424. doi: 10.1016/j.jmb.2012.02.002

Camilloni, C., De Simone, A., Vranken, W., and Vendruscolo, M. (2012). Determination of secondary structure populations in disordered states of proteins using NMR chemical shifts. Biochemistry 51, 2224-2231. doi: 10.1021/bi3001825

Cook, J. D., Bencze, K. Z., Jankovic, A. D., Crater, A. K., Busch, C. N., Bradley, P. B., et al. (2006). Monomeric yeast frataxin is an iron-binding protein. Biochemistry 45, 7767-7777. doi: 10.1021/bi060424r

Delaglio, F., Grzesiek, S., Vuister, G. W., Zhu, G., Pfeifer, J., and Bax, A. (1995). Nmrpipe-a multidimensional spectral processing system based on Unix pipes. J. Biomol. NMR 6, 277-293. doi: 10.1007/BF00197809

He, Y., Alam, S. L., Proteasa, S. V., Zhang, Y., Lesuisse, E., Dancis, A., et al. (2004). Yeast frataxin solution structure, iron binding, and ferrochelatase interaction. Biochemistry 43, 16254-16262. doi: 10.1021/bi0488193

Iannuzzi, C., Adrover, M., Puglisi, R., Yan, R., Temussi, P. A., and Pastore, A. (2014). The role of zinc in the stability of the marginally stable IscU scaffold protein. Protein Sci. 23, 1208-1219. doi: 10.1002/pro.2501

Johnson, B. A., and Blevins, R. A. (1994). NMRView, A computer program for the visualization and analysis of NMR data. J. Biomol. NMR 4, 603-614. doi: 10.1007/BF00404272

Karlberg, T., Schagerlöf, U., Gakh, O., Park, S., Ryde, U., Lindahl, M., et al. (2006). The structures of frataxin oligomers reveal the mechanism for the delivery and detoxification of iron. Structure 14, 1535-1546. doi: 10.1016/j.str.2006.08.010

Keller, R. L. J. (2004). The Computer Aided Resonance Assignment Tutorial 01/2004, 1st Edn. Goldau: CANTINA Verlag. ISBN: 3-85600-112-3.

Koradi, R., Billeter, M., and Wüthrich, K. (1996). MOLMOL, a program for display and analysis of macromolecular structure. J. Mol. Graph. 14, 51-55. doi: 10.1016/0263-7855(96)00009-4

Marsh, J. A., Singh, V. K., Jia, Z., and Forman-Kay, J. D. (2006). Sensitivity of secondary structure propensities to sequence differences between alpha- and gamma-synuclein, implications for fibrillation. Protein Sci. 15, 2795-2804. doi: 10.1110/ps.062465306

Martin, S. R., Esposito, V., De Los Rios, P., Pastore, A., and Temussi, P. A. (2008) The effect of low concentrations of alcohols on protein stability, a cold and heat denaturation study of yeast frataxin. J. Am. Chem. Soc. 130, 9963-9970. doi: 10.1021/ja803280e

Nair, M., Adinolfi, S., Pastore, C., Kelly, G., Temussi, P., and Pastore, A. (2004). Solution structure of the bacterial frataxin ortholog, CyaY, mapping the iron binding sites. Structure 12, 2037-2048. doi: 10.1016/j.str.2004.08.012

Pandolfo, M., and Pastore, A. (2009). The pathogenesis of Friedreich ataxia and the structure and function of frataxin. J. Neurol. 256 Suppl. 1, 9-17. doi: 10.1007/s00415-009-1003-2

Pastore, A., Martin, S. R., Politou, A., Kondapalli, K. C., Stemmler, T., and Temussi, P. A. (2007). Unbiased cold denaturation, low- and high-temperature unfolding of yeast frataxins under physiological conditions. J. Am. Chem. Soc. 129, 5374-5375. doi: 10.1021/ja0714538

Piotto, M., Saudek, V., and Sklenár, V. (1992). Gradient-tailored excitation for single-quantum NMR spectroscopy of aqueous solutions. J. Biomol. NMR 2, 661-665. doi: 10.1007/BF02192855

Qian, B., Raman, S., Das, R., Bradley, P., McCoy, A. J., Read, R. J., et al. (2007). High-resolution structure prediction and the crystallographic phase problem. Nature 450, 259-264. doi: 10.1038/nature06249

Ramelot, T. A., Raman, S., Kuzin, A. P., Xiao, R., Ma, L. C., Acton, T. B., et al. (2009). Improving NMR protein structure quality by Rosetta refinement, a molecular replacement study. Proteins 75, 147-167. doi: 10.1002/prot.22229 
Razvi, A., and Scholtz, J. M. (2006). Lessons in stability from thermophilic proteins. Protein Sci. 15, 1569-1578. doi: 10.1110/ps.062130306

Rimratchada, S., McLeish, T. C., Radford, S. E., and Paci, E. (2014). The role of high-dimensional diffusive search, stabilization, and frustration in protein folding. Biophys. J. 106, 1729-1740. doi: 10.1016/j.bpj.2014. 01.051

Sanfelice, D., Puglisi, R., Martin, S. R., Di Bari, L., Pastore, A., and Temussi, P. A. (2014). Yeast frataxin is stabilized by small salt concentrations, cold denaturation helps disentangling ionic strength effects from specific interactions PLoS ONE 9:e95801. doi: 10.1371/journal.pone.0095801

Sattler, M., Schleucher, J., and Griesinger, C. (1999). Heteronuclear multidimensional NMR experiments for the structure determination of proteins in solution employing pulsed field gradients. Prog. Nucl. Mag. Res. Sp. 34, 93-158. doi: 10.1016/S0079-6565(98)00025-9

Shen, Y., Lange, O., Delaglio, F., Rossi, P., Aramini, J. M., Liu, G., et al. (2008). Consistent blind protein structure generation from NMR chemical shift data. Proc. Natl. Acad. Sci. U.S.A. 105, 4685-4690. doi: 10.1073/pnas. 0800256105

Shen, Y., Delaglio, F., Cornilescu, G., and Bax, A. (2009). TALOS+, A hybrid method for predicting protein backbone torsion angles from NMR chemical shifts. J. Biomol. NMR 44, 213-223. doi: 10.1007/s10858-009-9333-z

Ternström, T., Mayor, U., Akke, M., and Oliveberg, M. (1999). From snapshot to movie, phi analysis of protein folding transition states taken one step further. Proc. Natl. Acad. Sci. U.S.A. 96, 14854-14859. doi: 10.1073/pnas.96. 26.14854
Wishart, D. S., Bigam, C. G., Yao, J., Abildgaard, F., Dyson, H. J., Oldfield, E., et al. (1995). ${ }^{1} \mathrm{H},{ }^{13} \mathrm{C}$ and ${ }^{15} \mathrm{~N}$ chemical shift referencing in biomolecular NMR. $J$. Biomol. NMR 6, 135-140. doi: 10.1007/BF00211777

Zong, C., Wilson, C. J., Shen, T., Wittung-Stafshede, P., Mayo, S. L., and Wolynes, P. G. (2007). Establishing the entatic state in folding metallated Pseudomonas aeruginosa azurin. Proc. Natl. Acad. Sci. U.S.A. 104, 3159-3164. doi: 10.1073/ pnas.0611149104

Conflict of Interest Statement: The authors declare that the research was conducted in the absence of any commercial or financial relationships that could be construed as a potential conflict of interest.

Received: 30 July 2014; paper pending published: 27 August 2014; accepted: 11 September 2014; published online: 30 September 2014.

Citation: Vilanova B, Sanfelice D, Martorell G, Temussi PA and Pastore A (2014)

Trapping a salt-dependent unfolding intermediate of the marginally stable protein Yfh1. Front. Mol. Biosci. 1:13. doi: 10.3389/fmolb.2014.00013

This article was submitted to Structural Biology, a section of the journal Frontiers in Molecular Biosciences.

Copyright (c) 2014 Vilanova, Sanfelice, Martorell, Temussi and Pastore. This is an open-access article distributed under the terms of the Creative Commons Attribution License (CC BY). The use, distribution or reproduction in other forums is permitted, provided the original author(s) or licensor are credited and that the original publication in this journal is cited, in accordance with accepted academic practice. No use, distribution or reproduction is permitted which does not comply with these terms. 